\title{
"It's Time to Represent": shifting the paradigm to improve the quality of inputs into value assessment frameworks
}

Leticia R Moczygemba, PharmD, PhD; Carolyn Brown, PhD; and Michael Johnsrud, PhD, RPh

\section{ABSTRACT}

"It's Time to Represent" integrates 2 strategies that challenge the status quo to increase the diversity of populations that participate in research and address drivers of health disparities to better inform value assessment. The first, a community-engaged campaign, proposes to develop authentic, long-term partnerships with community members, their health care providers, and researchers to tailor recruitment and retention methods for underrepresented groups and hold researchers accountable for equitable selection of study participants. The second proposes to create an expectation for researchers to routinely collect patient-reported, actionable social determinants of health data to generate enhanced real-world evidence and thereby improve the quality of inputs utilized in value assessment frameworks.
"It's Time to Represent" is a 2-pronged strategy to increase the diversity of populations that participate in research and address drivers of health disparities to better inform value assessment (Figure 1) with the following objectives: (1) launch a comprehensive national campaign using a community-engaged approach to inform, create buy-in, and generate excitement for participation in research, and (2) enhance information used in value assessment frameworks by expediting current methodological initiatives to require a minimum set of patient-reported social determinants of health (SDOH) elements to be collected and reported in research, including clinical trials and observational (realworld) studies.

\section{Community-Engaged Campaign to Increase Representation}

The evidence hierarchy used in value frameworks leaves large gaps in the ability to translate value when the evidence base used does not represent the economic, clinical, or patientcentric diversity of the populations that are impacted by the interventions themselves. Authentic participation and representation in research require a paradigm shift from current norms to meet underrepresented populations "where they are" in the community. Low trust, high distrust, high mistrust, and, simply, lack of opportunity are key reasons for minimal participation in research by these groups. "It's Time to Represent" recognizes that mistrust and distrust of "the system" by ethnic minorities and

\section{Author affiliations \\ Leticia R Moczygemba, PharmD, PhD; Carolyn Brown, PhD; and Michael Johnsrud, $\mathrm{PhD}, \mathrm{RPh}$, Health Outcomes Division and Texas Center for Health Outcomes Research \& Education (TxCORE), College of Pharmacy, University of Texas at Austin.}

AUTHOR CORRESPONDENCE: Leticia R Moczygemba, 512.232.6880;

Irmoczygemba@austin.utexas.edu

$J$ Manag Care Spec Pharm. 2021;27(9-a):S17-S21

Copyright $\odot 2021$, Academy of Managed Care Pharmacy. All rights reserved.

other disadvantaged groups are reasonable and valid responses to their past and present experiences and that any successful approach should also acknowledge that "the system" needs to demonstrate it is indeed trustworthy. ${ }^{1}$ Thus, a grassroots effort with a different kind of appeal is needed to galvanize community members to evoke change as true partners, rather than subjects, to reshape views about research roles and contributions to the health of their community.

Not unlike a comprehensive "Get Out the Vote" campaign, our "It's Time to Represent" strategy is multipronged and community-based. Community 


\section{FIGURE 1 "It's Time to Represent": Strategy Impact on Inputs to Inform Value}

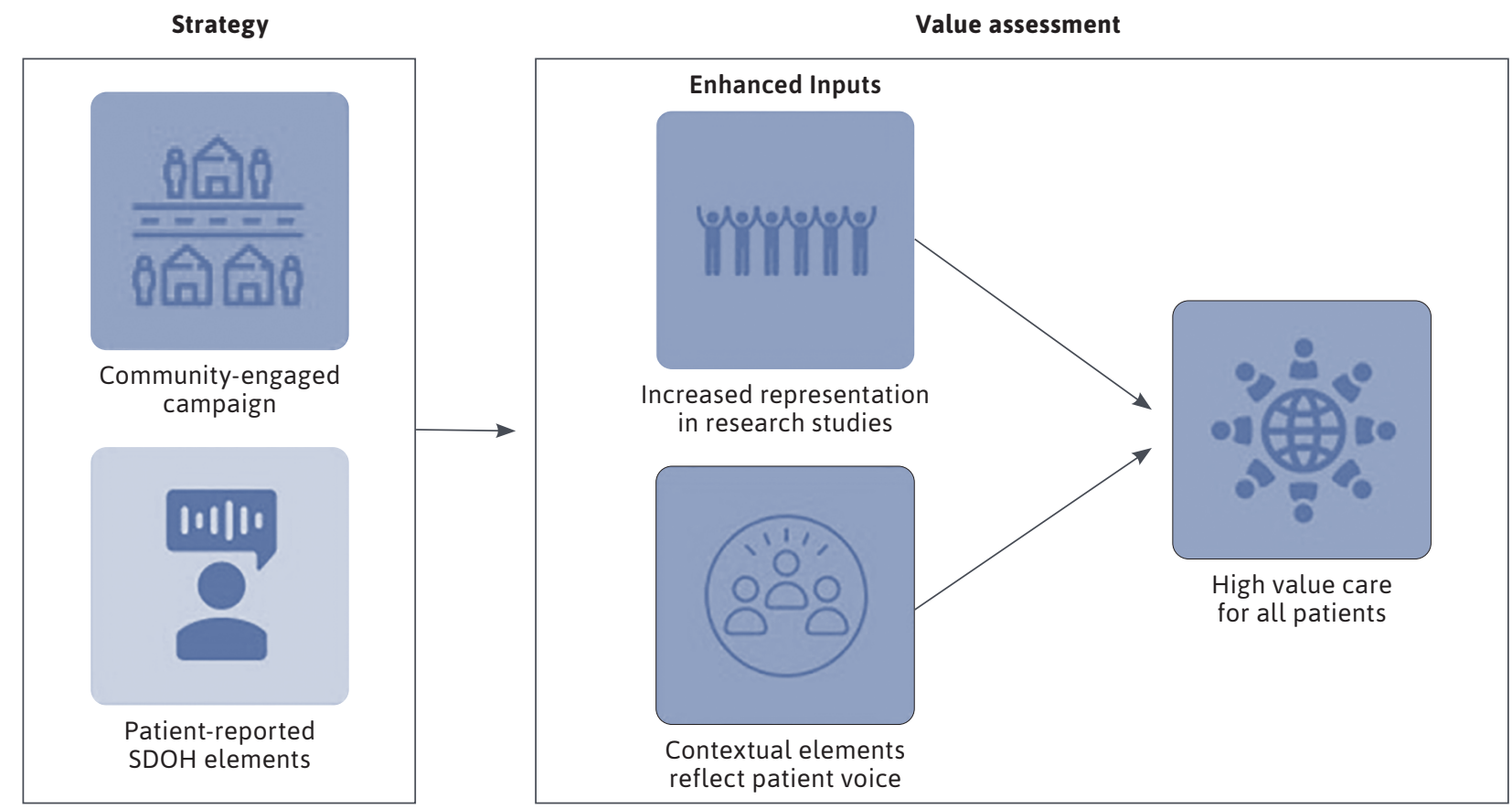

$\mathrm{SDOH}=$ social determinants of health.

members, their health care providers, and researchers should all be meaningfully engaged to increase the capacity to facilitate research participation and create communityinformed best practices. Trust issues and lack of access to information are common barriers to research participation across ethnic-minority groups; however, altruistic appeals rooted in cultural and community priorities are mutual facilitators in research participation among these groups. ${ }^{2}$

Evidence shows that communities need to be engaged before and after study recruitment to legitimately increase representation and retention of underrepresented groups. $^{3-5}$ Although this may seem straightforward, to do this in a meaningful way, researchers and funders must shift their thinking from subject, which is transactional and time-limited in that once study activities are complete the interaction is complete, to participant, which implies an active role as a partner. ${ }^{4}$ Getrich et al describes a cycle of trust whereby a relationship is being continuously cultivated before the study, during recruitment, throughout the study, and after study completion. ${ }^{3}$ Initially, the research team can build trust by communicating effectively (eg, bilingual team members), demonstrating respect and understanding of cultural norms, developing an understanding of motivations for participation in research (eg, personal and/ or community benefits, contributing to the greater good), and including community representation on the team. ${ }^{3,5,6}$

Consensus building and developing shared goals for research also create a solid foundation of engagement and can lead to long-term partnerships., ${ }^{4,7}$ Investing the time before a study to learn about the community of focus will allow researchers to personalize recruitment and retention methods to include contextual factors relevant to a community and make potential participants feel like "more than a number." ${ }^{3,4}$ Equally important is continued interaction with the community upon study completion. Historically, "community-placed" research, where researchers have conducted research with no community involvement in an "up and out" fashion, has harbored deep resentment and decreased motivation of underrepresented groups to participate in research which in some cases may still exist today. ${ }^{8}$ Thus, strategies such as sharing study findings, co-ownership of data, and holding community forums for feedback can be used to stay engaged with communities once study participation is completed. ${ }^{4,7}$ To move up the ladder of participation from nonparticipation to empowered participants, commitment in the form of time 
and other resources is required on the part of researchers, communities, and funders. ${ }^{79}$ The Patient Centered Outcomes Research Institute is an exemplar in upholding rigorous standards for patient engagement in research, even including patient stakeholders in funding decisions. A similar model can be used by funders and other stakeholders to ensure that authentic engagement occurs.

In parallel, researchers should be accountable to ensuring their research designs include equitable selection-without loopholes-of people who are most impacted by the outcomes being studied. As exemplified by the guidance recently released by the US Food and Drug Administration on improving diversity in clinical trials, ${ }^{10}$ and Pharmaceutical Research and Manufacturers of America's newest principle of "enhancing diversity in clinical trial participation," which has been added to the Principles on Conduct of Clinical Trials and Communication of Clinical Trial Results, ${ }^{11}$ it is critical that diverse groups be better represented in research. Often, the design of clinical trials systematically excludes the people in which they were designed to help. For example, stringent inclusion/exclusion criteria, employment constraints, cultural differences, and implementation in academic centers that may not be accessible to diverse groups are known and ongoing impediments to participation among ethnic minorities that must be addressed. ${ }^{3,12,13} \mathrm{~A}$ recent meta-analysis revealed that, when asked, Black patients participated in cancer clinical trials at similar rates as White patients. ${ }^{14}$ The authors noted, and we agree, that attention to modifiable structural (eg, patient access to available trials, provider resources/infrastructure for participation) and clinical (eg, broadening eligibility criteria) barriers could improve trial

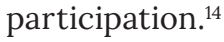

Providers who are people of color need to be at the forefront of any strategy to increase research participation of their patients..$^{15}$ The creation of a central repository of providers who serve communities of color, as well as other medical and health practices, and community-based organizations where people of color are cared for and accessible is a key element of this initiative. Many ethnic minority physicians would likely participate in clinical trials if trial designs were modified to accommodate their resource level and reduce participant burden. ${ }^{12}$

Doctors, pharmacists, other health care providers, and other community-based champions should be provided the protocol information, training, and tools to inform, engage, and activate patients and community members while building their trust, interest, and ability to participate in research studies. Implementation should be flexible and nimble because training, information, and resource needs may vary based on research experience, which will expectedly be limited among those who primarily serve communities of color. Ultimately, investigators will have access to this rich, centralized resource for the recruitment of eligible and willing participants into clinical trials and observational research studies.

\section{Enhancing Value Assessment Through Consideration of Social Determinants of Health Data}

The approach we outline for better representation of diverse voices in value assessment frameworks targets the sources of evidence within these frameworks, namely randomized controlled trials (RCTs) and observational (real-world) data. Our "Get Out the Vote" approach is primarily directed at increasing participation in RCTs, which are often the primary source of data cited from the evidence base to make clinical and cost-effectiveness determinations that produce the judgment of a medication's value. Therefore, systems that facilitate recruitment of underrepresented patient populations into RCTs are an important mechanism to generate the needed data and evidence to better inform the value modeling processes used within most frameworks in the United States today.

In addition, an important gap within the evidence base that can provide more robust context for health equity is the integration of social determinants of health (SDOH) data into value frameworks through its collection at the point of care as observational, or real-world, data. Our approach addresses this key shortcoming in that whole-person contextual inputs are currently inadequate in current value assessment frameworks because clinical and cost-effectiveness studies that inform value determination nearly always lack appropriate risk adjustment for meaningful SDOH factors in the population. This leads to biased results that impact the interpretation of value when attempting to generalize to patient populations with higher $\mathrm{SDOH}$ needs. Therefore, SDOH data as a potential contributor to more robust real-world data analyses should not be overlooked. As an example, recent research has established that Medicare Advantage quality rankings related to diabetes and cholesterol control would have increased for up to 1 in 5 plans if risk adjustment that incorporated sociodemographic factors across disadvantaged populations was considered..$^{16}$

The generation of real-world evidence that incorporates $\mathrm{SDOH}$ can be an important source of data to inform value frameworks. Recent research indicates that although the use of real-world evidence in a value framework such as the Institute for Clinical and Economic Review (ICER) has 
varied in its application across the organization's published reports, ICER has provided guidance that describes opportunities for use of real-world evidence to inform coverage and formulary decisions. ${ }^{17,18}$

However, our ability to leverage $\mathrm{SDOH}$ data within value frameworks is currently hindered by the fact that the methodologies to collect and integrate this contextual data has not fully matured, particularly patient-reported $\mathrm{SDOH}$ data. We must expedite our development of standards for $\mathrm{SDOH}$ data collection and further incentivize the use of $\mathrm{SDOH}$ in decision making through integration into quality and performance measures to expand available data for use in real-world evidence generation, which complements RCT data in value assessment frameworks. Integration into performance and quality measures will further serve as incentives for establishment and maintenance of standards for collecting SDOH data. The incentives will also create a proliferation of $\mathrm{SDOH}$ data generation that can provide the additional data elements needed for real-world evidence analyses that incorporate appropriate risk-adjustment, as described earlier. In light of this, a recent policy framework was announced by the US House Ways and Means Committee that seeks to address gaps in health equity by prioritizing approaches to standardization of metrics to measure health equity, as well as further definition of SDOH. ${ }^{19}$

A further challenge is that existing efforts to measure $\mathrm{SDOH}$ in research often rely upon surrogate markers (eg, ZIP code, income) that are imprecise and without the patient voice. These markers are chosen largely out of availability in current data sets, but "settling" for this approach to identifying gaps in SDOH lacks specificity and, ultimately, utility. The creation and validation of a minimum set of patient-reported SDOH elements will begin to close the gap and address currently unmeasured confounders that exist in studies that inform value assessments.

$\mathrm{SDOH}$ data should be collected with the intent to be actionable. This requires pairing its collection with the meaningful development of mechanisms to actually address gaps at the individual level through the coordination of available community services by providers at the point of care. Recent guidance from the Centers for Medicare \& Medicaid Services has provided a potential roadmap and examples for state Medicaid programs to broaden availability of, and access to, services to address gaps at the community level. ${ }^{6}$ While policymakers continue to deliberate on the structure and funding required to support these programs, this should not impede our advancement toward collection of patient-reported $\mathrm{SDOH}$ data; rather, it is a call to incentivize its collection in order to better identify where near-term resources can best address prioritized gaps in patient populations most effectively.

A multistakeholder approach (ie, patients, community representatives, clinicians, researchers, funders) should build on early initiatives such as the Gravity Project to continue to develop pathways for broader collection of $\mathrm{SDOH}$ data elements, and go further to instill an expectation for researchers to include $\mathrm{SDOH}$ in their study design and analyses. The focus on incorporation of $\mathrm{SDOH}$ enhances the richness and perspective of data and evidence used by all value assessment frameworks, regardless of therapeutic focus. The result is better informed and more equitable decisions of value being made across diverse groups and mitigation of the harmful effects of the current "one size fits all" approach.

\section{It's Time to Represent}

Our body of evidence is in dire need of better representation from diverse patient populations. The proposed 2-pronged strategy is a bold approach using authentic community engagement to increase diversity in clinical research, paired with expedited data infrastructure development that collects much needed patient-reported $\mathrm{SDOH}$ elements as part of real-world evidence generation, which will create a "voice" to that body of evidence. Unfortunately, this voice has been previously much too faint. Giving clear voice to the body of evidence as we move forward more authentically informs how value is determined.

Let's begin with this bold plan: It's Time to Represent!

\section{DISCLOSURE}

No specific funding was received for this manuscript. The authors report no potential conflicts of interest.

\section{REFERENCES}

1. Griffith DM, Bergner EM, Fair AS, Wilkins $\mathrm{CH}$. Using mistrust, distrust, and low trust precisely in medical care and medical research advances health equity. Am J Prev Med. 2020;60(3):442-45.

2. George S, Duran N, Norris K. A systematic review of barriers and facilitators to minority research participation among African Americans, Latinos, Asian Americans, and Pacific Islanders. Am J Public Health. 2014;104(2):e16-e31.

3. Getrich CM, Sussman AL, CampbellVoytal K, et al. Cultivating a cycle of trust with diverse communities in practicebased research: a report from PRIME Net. Ann Fam Med. 2013;11(6):550-58.

4. Bromley E, Mikesell L, Jones F, Khodyakov D. From subject to participant: ethics and the evolving role of community in health research. Am J Public Health. 2015;105(5):900-08. 
5. Woolf SH, Zimmerman E, Haley A, Krist AH. Authentic engagement of patients and communities can transform research, practice, and policy. Health Aff (Millwood). 2016;35(4):590-94.

6. Brunton G, Thomas J, O'Mara-Eves A, Jamal F, Oliver S, Kavanagh J. Narratives of community engagement: a systematic review-derived conceptual framework for public health interventions. BMC Public Health. 2017;17(1):944.

7. London JK, Haapanen KA, Backus A, Mack SM, Lindsey M, Andrade K. Aligning community-engaged research to context. Int J Environ Res Public Health. 2020;17(4):1187.

8. Damon W, Callon C, Wiebe L, Small W, Kerr T, McNeil R. Community-based participatory research in a heavily researched inner city neighbourhood: perspectives of people who use drugs on their experiences as peer researchers. Soc Sci Med. 2017;176:85-92.

9. Arnstein SR. A ladder of citizen participation. J Am Inst Plann. 1969;35(4):216-24.

10. US Department of Health and Human Services, Food and Drug Administration, Center for Drug Evaluation and Research, Center for Biologics Evaluation and Research. Enhancing the diversity of clinical trial populations: eligibility criteria, enrollment practices, and trial designs. Guidance for Industry. November 2020. Accessed June 2, 2021. https://www.fda. gov/media/127712/download
11. Pharmaceutical Research and Manufacturers of America. Principles on conduct of clinical trials communication of clinical trial results. October 14 , 2020. Accessed June 2, 2021. https:// phrma.org/-/media/Project/PhRMA/ PhRMA-Org/PhRMA-Org/PDF/P-R/ PhRMAPrinciples-of-Clinical-TrialsFINAL.pdf

12. Salman A, Nguyen C, Lee YH, Cooksey-James T. A review of barriers to minorities' participation in cancer clinical trials: Implications for future cancer research. J Immigr Minor Health. 2016;18(2):447-53.

13. Vaswani PA, Tropea TF, Dahodwala N. Overcoming barriers to Parkinson disease trial participation: increasing diversity and novel designs for recruitment and retention. Neurotherapeutics. 2020;17 (4):1724-35.

14. Unger JM, Hershman DL, Till C, et al. "When offered to participate": a systematic review and meta-analysis of patient agreement to participate in cancer clinical trials. J Natl Cancer Inst. 2021;113(3):244-57.

15. Bajaj SS, Stanford FC. Beyond Tuskegee-vaccine distrust and everyday racism. N Engl J Med. 2021;384(5):e12.
16. Durfey SNM, Kind AJH, Gutman R, et al. Impact of risk adjustment for socioeconomic status on Medicare Advantage plan quality rankings. Health Aff (Millwood). 2018;37(7):1065-72.

17. Lee W, Dayer V, Jiao B, Carlson JJ, Devine B, Veenstra DL. Use of real-world evidence in economic assessments of pharmaceuticals in the United States. J Manag Care Spec Pharm. 2021;27(1):5-14. doi: 10.18553/jmcp.2021.27.1.005

18. Pearson SD, Dreitlein WB, Towse A, Hampson G, Henshall C. A framework to guide the optimal development and use of real-world evidence for drug coverage and formulary decisions. J Comp Eff Res. 2018;7(12);1145-52.

19. US House Committee on Ways and Means. Policy pillars and priorities: a bold vision for a legislative pathway toward health and economic equity. January 11, 2021. Accessed June 2, 2021. https://waysandmeans.house.gov/sites/democrats. waysandmeans.house.gov/files/documents/FINAL\%20WMD\%20Health\%20 and\%20Economic\%20Equity $\% 20$ Vision\%20Policy_FRAMEWORK.pdf 